\title{
Effects of human activities on rivers located in protected areas of the Atlantic Forest
}

\author{
Efeitos de atividades antrópicas em rios localizados em Unidades \\ de Conservação de Mata Atlântica
}

Mônica Luisa Kuhlmann ${ }^{1}$, Hélio Rubens Victorino Imbimbo ${ }^{1}$, Lucy Lina Ogura ${ }^{1}$,

João Paulo Villani ${ }^{2}$, Roberto Starzynski ${ }^{3}$ and Maria de Jesus Robim ${ }^{3}$

${ }^{1}$ Companhia Ambiental do Estado de São Paulo - CETESB, Av. Prof. Frederico Hermann Jr, 345, CEP 05459-900, São Paulo, SP, Brazil

e-mail: mkuhlmann@sp.gov.br; himbimbo@sp.gov.br; logura@sp.gov.br ${ }^{2}$ Núcleo Santa Virgínia do PESM, Fundação Florestal, Rod. Dr. Oswaldo Cruz, Km 78, CEP 12140-000, São Luís do Paraitinga, SP, Brazil e-mail: jpvillani@hotmail.com

${ }^{3}$ Instituto Florestal, Divisão de Reservas e Parques Estaduais, Rua do Horto, 931, CEP 02377-000, São Paulo, SP, Brazil

e-mail: rostarzynski@hotmail.com; mjesusrobim@gmail.com

\begin{abstract}
Aim: This study evaluated the impacts of anthropogenic activities upstream of conservation areas on the Paraibuna river and its implications for freshwater biodiversity. Methods: The study was carried out in two units, Cunha and Santa Virginia, of the Serra do Mar State Park (SP), located in the Atlantic Rain Forest. Five sampling sites were defined, four along the Paraibuna river and one in the Ipiranga river, the latter fully inserted into the protected area. Physical, chemical, microbiological and ecotoxicological data were obtained from surface water as well as aquatic macroinvertebrates. Results: The results showed that the waters of the Paraibuna river have low anthropogenic interference. However, conductivity, turbidity, coliforms, iron, total phosphorus and nitrate showed a gradient improving its water quality from upstream to downstream, indicating the existence of erosion and introduction of organic debris in the basin. The BMWP index, varying from 58 to 190 , also showed the good condition of the river to aquatic biota, with predominant Excellent quality diagnosis. The values of this index and the richness index $(\mathrm{S})$ outlined a similar gradient but with the lowest values recorded in P3. Conclusions: The results showed that the upstream activities alter the natural condition of the Paraibuna river and its biota and that the protected areas provides environmental services reducing these impacts. The ideal situation in order to ensure the conservation of the freshwater biota of the Paraibuna river would be the incorporation of parts of the upstream area into the protected area and convert occupied areas into Sustainable Use Area, that guarantee the adoption of sustainable techniques to the existing land uses and the application of aquatic life protection indicators for monitoring the water quality of the river.
\end{abstract}

Keywords: Paraibuna river, Serra do Mar State Park, impact, protected area, macroinvertebrates.

Resumo: Objetivo: Este trabalho avaliou os impactos de usos antrópicos sobre o rio Paraibuna em área situada à montante de unidades de conservação e sua implicação para a conservação de ecossistemas límnicos. Métodos: $\mathrm{O}$ estudo foi desenvolvido no Parque Estadual da Serra do Mar (SP), inserido no domínio da Mata Atlântica, em dois de seus núcleos: Cunha e Santa Virgínia. Foram estabelecidos cinco pontos de coleta, quatro no rio Paraibuna e um no rio Ipiranga, este último totalmente inserido na unidade de conservaçáo. Nestes locais foram obtidos dados físicos, químicos, microbiológicos e toxicológicos da água superficial e de macroinvertebrados aquáticos. Resultados: Os resultados mostraram que as águas do rio Paraibuna sofrem baixa interferência antrópica. Contudo, as variáveis condutividade, turbidez, coliformes, $\mathrm{Fe}, \mathrm{P}_{\text {tot }}$ e nitrato evidenciaram a existência de um gradiente de qualidade no sentido montante - jusante, apontando a existência de problemas de erosão e introdução de despejos orgânicos na bacia. O índice BMWP, com valores entre 58 a 190, também evidenciou a boa condição do rio para a biota aquática, prevalecendo o diagnóstico de qualidade excelente. No entanto, os valores deste índice e da riqueza 
(S) também retrataram um gradiente similar ao dos dados ambientais. Conclusóes: Os resultados obtidos mostraram que as atividades a montante da unidade de conservação modificam as condiçóes naturais do rio Paraibuna e, consequentemente, sua biota e que a unidade de conservaçáo presta serviço ambiental ao amortizar estes impactos. Para que se assegure a conservação da biota do rio Paraibuna seria ideal a incorporaçáo de parte da área a montante pela UC e a transformação da área antropizada em Unidades de Conservação de Uso Sustentável que assegure, por seus instrumentos de gestáo, a adoção de melhores técnicas para os usos de solo existentes e a aplicação de indicadores ecológicos no monitoramento de qualidade da água do rio.

Palavras-chave: rio Paraibuna, Parque Estadual da Serra do Mar, impacto, unidade de conservação, macroinvertebrados.

\section{Introduction}

The biodiversity in ecosystems provides resistance and resilience to disturbance (Vinson and Hawkins, 1998). Riparian environments have high species diversity, which have a strong tendency to endemism since they are naturally isolated within a basin or even in smaller areas (Allan and Flecker, 1993). However, these ecosystems are probably the most threatened by anthropic activities and the conservation of their species has rarely been the subject of the creation of protected areas, especially those intended for strict protection. In Brazil, the conservation areas created in the region of Pantanal and Bonito are exceptions. Official lists of endangered species already include elements of aquatic ecosystems fauna and flora (Brasil, 2003, 2004; São Paulo, 2004, 2010) and as the anthropogenic pressures on these ecosystems get more intense, the risk of extinction increases (Strayer, 2006). Rivers and streams are strongly influenced by the landscape in which they are inserted (Allan, 2004), so in the conservation of freshwater species it is necessary to consider the whole drainage basin, and in some cases, a larger area might be required (Strayer, 2006).

In Brazil, protected areas are separated in two types (Brasil, 2000): the Strict Protection Area, which has the purpose of nature conservation, without direct use of its resources; and the Sustainable Use Area, which must reconcile conservation and sustainable use of natural resources.

São Paulo state has the largest continuous area of Strict Protection Area of Atlantic Rain Forest, formed by the Serra do Mar State Park. Created in 1977 with educational, recreational and scientific purposes (São Paulo, 2006), it currently comprises an area of $3,326.8 \mathrm{~km}^{2}$ divided into nine units, each with its own manager.

The Serra do Mar State Park protects a number of small streams that run entirely within the protected area, but also receives some watercourses that rise upstream, outside its boundaries, which can be subjected to the impacts of human activities. That is the case of the Paraibuna river that has its headwaters located upstream of the Cunha unit, outside the protected area. In this region there are small farms, with animal husbandry (chicken, cattle, sheep, trout), agriculture and eucalyptus plantations. Moreover, along its course, the streams that drain into the Paraibuna river have their margins subjected to cattle trampling, which is facilitated by the absence of riparian vegetation and also prevents its natural regeneration process. There are also impacts associated to the existence of rural roads bordering the Paraibuna river or its tributaries that are used as an access to neighborhoods and to the park itself.

The Paraibuna river, after $163 \mathrm{~km}$ long, joint to the Paraitinga river to form the Paraíba do Sul river that flows northeast towards the state of Rio de Janeiro to empty into the Atlantic Ocean. Its basin is inserted in the Paraíba Valley, a highly urbanized and industrialized region of São Paulo state. Municipalities from São Paulo and Rio de Janeiro states use the waters of the basin for different purposes, including power generation, irrigation, public and industrial supply.

Aquatic macroinvertebrates are the most used biota for freshwater biomonitoring worldwide (Rosenberg and Resh, 1993). Its data allow to assess if the environment quality favors or not the local biodiversity and therefore is useful for evaluating preventive possible damage to preserved environments, such as water bodies on conservation units.

This study aimed to evaluate possible impacts caused by human activities in the headwaters of the Paraibuna river, which flows through the Serra do Mar State Park, and to discuss adequate actions for biomonitoring and conservation of aquatic biota of inland waters in similar situations. 


\section{Material and Methods}

\subsection{Study area}

Paraibuna means big river of dark waters (Pará = big river; $\mathrm{Y}=$ water; Una $=$ dark) in tupiguarani language, an indigenous tribe of the region. The river rises in Cunha city, located 1,600 m altitude, at Campo Alegre neighborhood and runs across two units of the Serra do Mar State Park, Cunha and Santa Virgínia, which have, respectively, 140 and $175 \mathrm{~km}^{2}$ (Såo Paulo, 2013). Its headwater is outside the boundaries of the Serra do Mar State Park and the river reaches the Cunha unit after $25.3 \mathrm{~km}$ as a sixth order body water, continuing with this classification through the entire course within the park.

At Santa Virginia unit runs the Ipiranga river, a fourth order watercourse with about $10 \mathrm{~m}$ wide. Fully inserted in the protected area, it was chosen as a reference area to generate optimal values for the analyzed parameters.

\subsection{Sampling and analytical procedures}

Five sampling sites were defined to assess the impacts on the Paraibuna river water quality of anthropogenic activities upstream and outside the limits of the Serra do Mar State Park (Figure 1):
P1 - Paraibuna river, Santa Virginia unit, Salto Grande, $23^{\circ} 20.718^{\prime} S-45^{\circ} 09.401^{\prime} \mathrm{W}$;

P2 - Paraibuna river, Santa Virginia unit, $23^{\circ} 19.877^{\prime} \mathrm{S}-45^{\circ} 08.481^{\prime} \mathrm{W}$;

P3 - Paraibuna river, Santa Virginia unit, Lajeado, $23^{\circ} 18.946^{\prime} \mathrm{S}-45^{\circ} 07.400^{\prime} \mathrm{W}$;

P4 - Paraibuna river, Cunha unit, Paraibuna river trail, $23^{\circ} 14.078^{\prime} \mathrm{S}-45^{\circ} 01.452^{\prime} \mathrm{W}$;

P5 - Ipiranga river, Santa Virginia unit, $23^{\circ} 20.939^{\prime} \mathrm{S}-45^{\circ} 08.143^{\prime} \mathrm{W}$.

Samplings were carried out in waterfalls sections (P1 and P4) or in the margins of the river (P2, P3 and P5) from April/09 to July/12 (Table 1).

Daily rainfall data were obtained using a recording rain gauge installed at the $\mathrm{D}$ Basin, near to the sampling site in the Cunha unit (P4), from January 2009 to December 2012.

Physical, chemical, microbiological and ecotoxicological analysis were made to establish the environmental gradient of the Paraibuna river. Measurements of conductivity, temperature, dissolved oxygen and $\mathrm{pH}$ were made in the field (electrometric method). Samples of surface water were taken for analysis of the following variables: turbidity (nephelometric method), dissolved solids (gravimetric method), alkalinity (titration method), faecal coliform (membrane

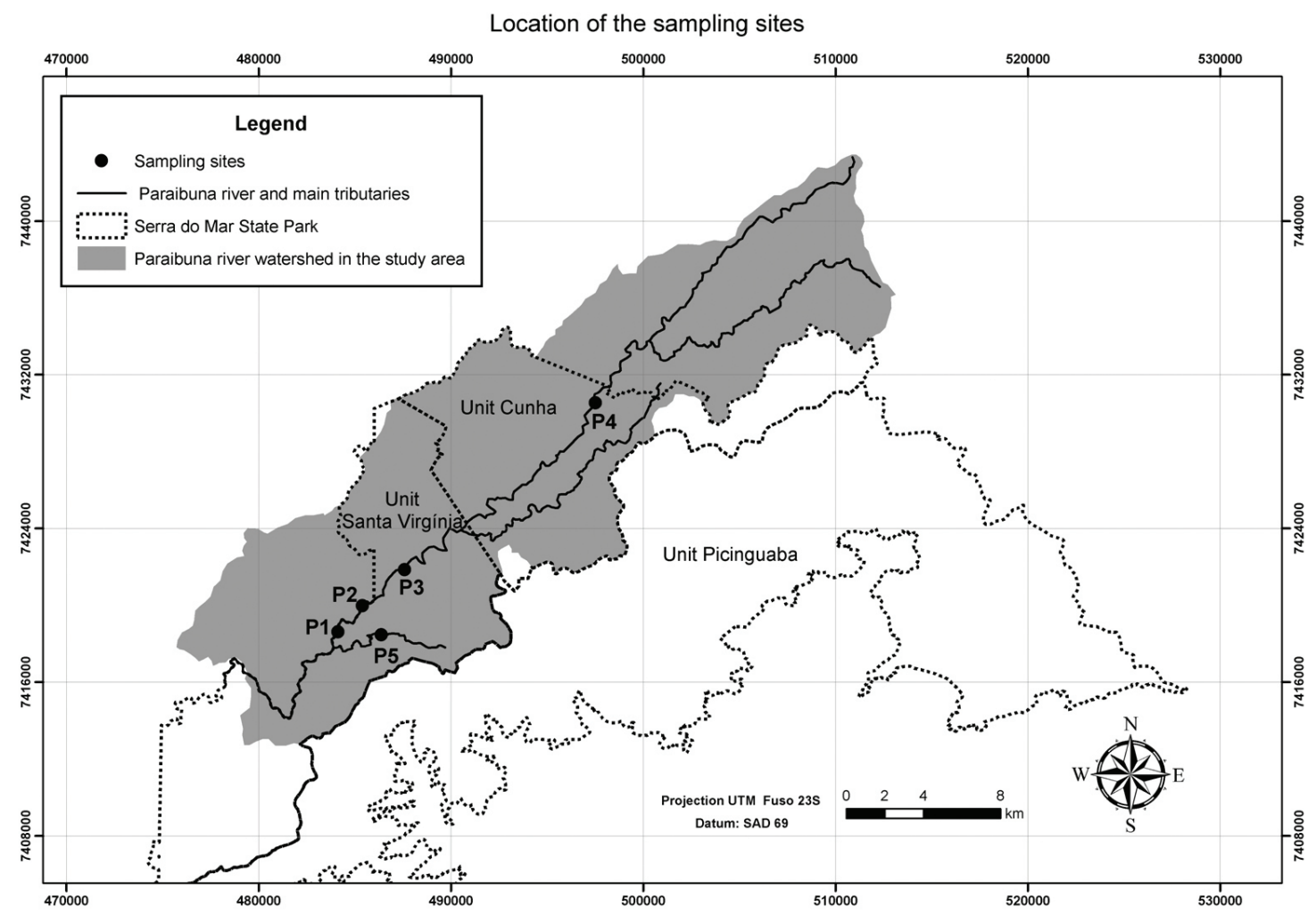

Figure 1. Sampling sites. 


\begin{tabular}{|c|c|c|c|}
\hline 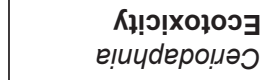 & 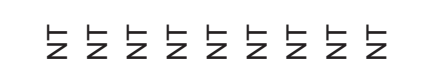 & 乞泣々乞 & 々 0 々 々々 々 \\
\hline 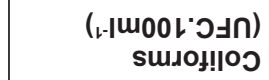 & 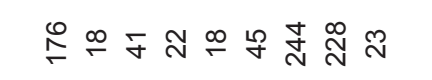 & 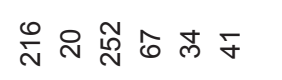 & \& \\
\hline 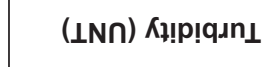 & 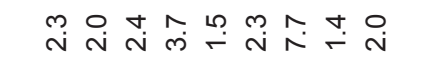 & 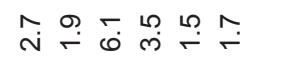 & 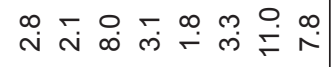 \\
\hline 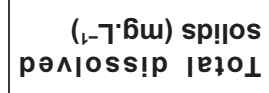 & 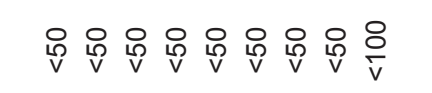 & 吕 & 吕 吕 \\
\hline 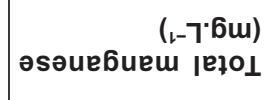 & 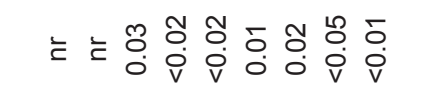 & 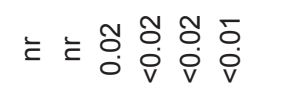 & 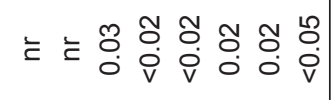 \\
\hline 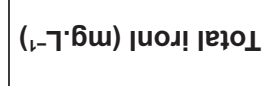 & 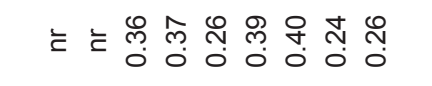 & 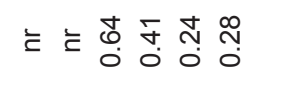 & 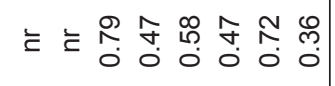 \\
\hline 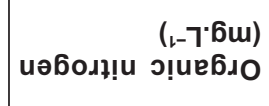 & 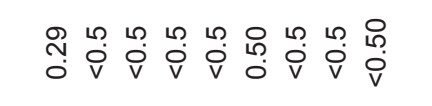 & 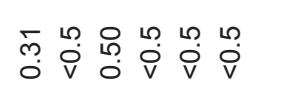 & 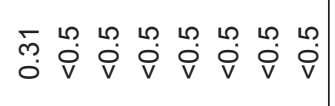 \\
\hline 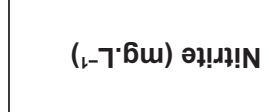 & 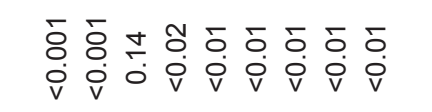 & 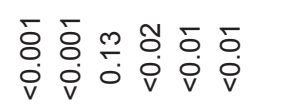 & 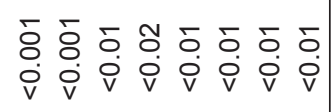 \\
\hline (^-7'6ul) әұеג!!N & 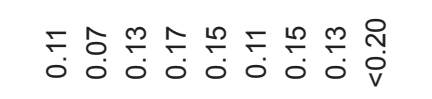 & 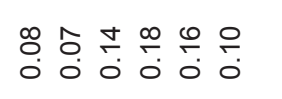 & 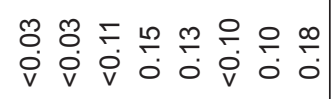 \\
\hline 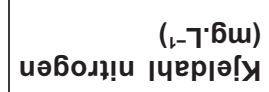 & 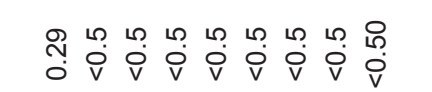 & 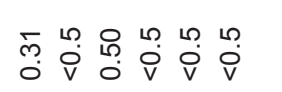 & 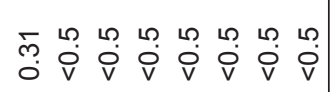 \\
\hline 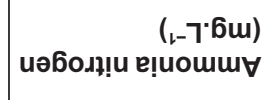 & 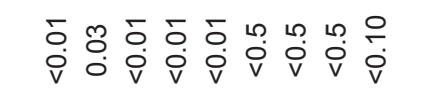 & 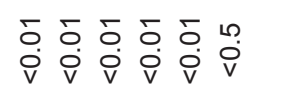 & 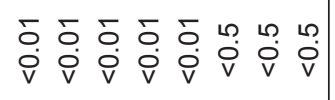 \\
\hline 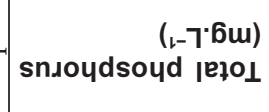 & 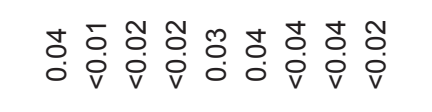 & 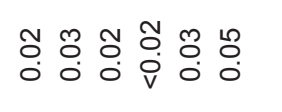 & 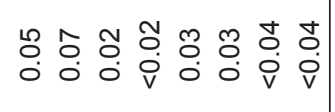 \\
\hline $\begin{array}{r}(1-7 \cdot 6 m) \\
\text { әңеydsoydoyม }\end{array}$ & 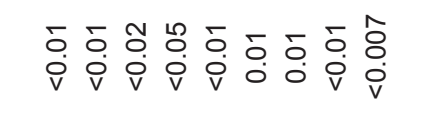 & 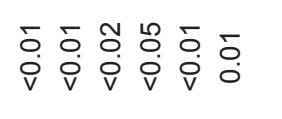 & 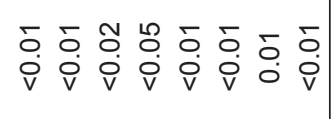 \\
\hline 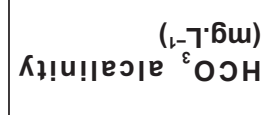 & 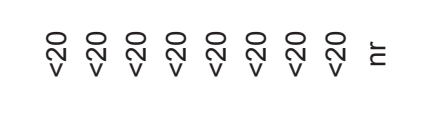 & 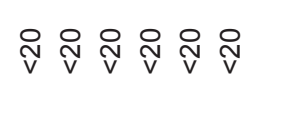 & 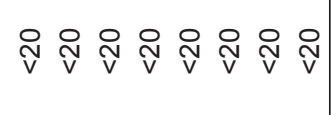 \\
\hline 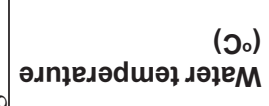 & 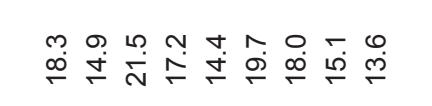 & 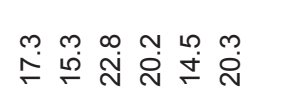 & 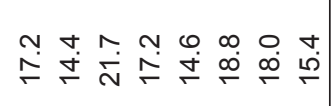 \\
\hline $\mathrm{Hd}^{\mathrm{d}}$ & 웃 & 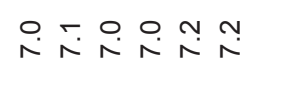 & テ オ \\
\hline $\begin{array}{r}\left(1-7^{\prime} \cdot 6 m\right) \\
\text { иәбКхо рәл|оss! }\end{array}$ & 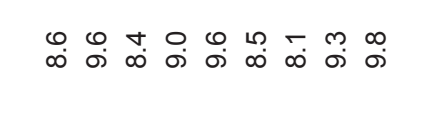 & 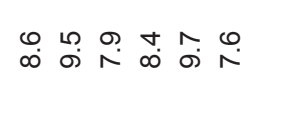 & $\begin{array}{llllllll}\infty & 0 & \infty & 0 & \infty & 0 & \infty & m \\
\infty & 0 & \infty & \oplus & \oplus & \infty & \infty & 0\end{array}$ \\
\hline 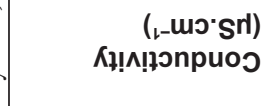 & 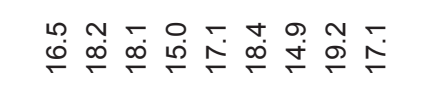 & 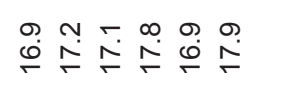 & 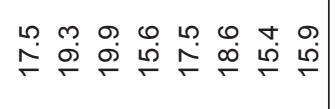 \\
\hline әңер & 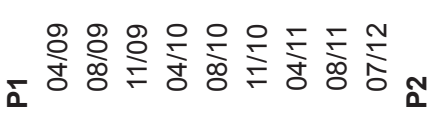 & 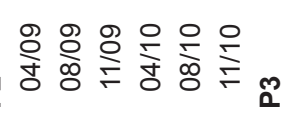 & 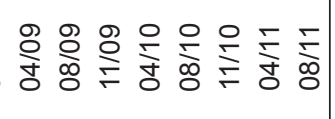 \\
\hline
\end{tabular}




\begin{tabular}{|c|c|c|}
\hline 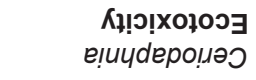 & 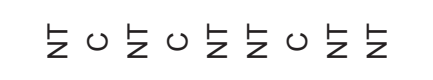 & 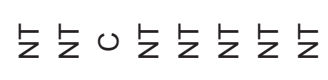 \\
\hline 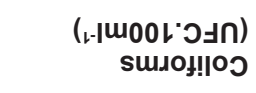 & 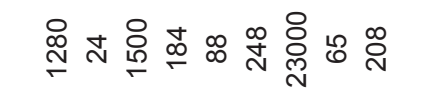 & 五 N \\
\hline 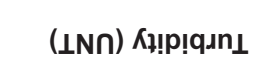 & 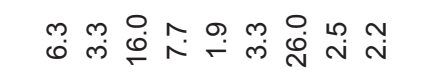 & 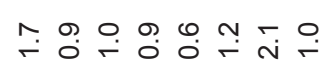 \\
\hline 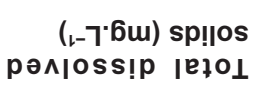 & 员 & 官 \\
\hline 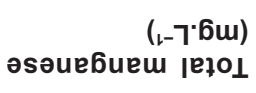 & 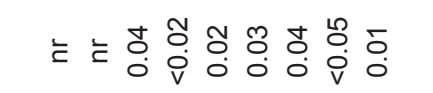 & 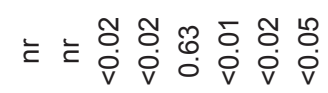 \\
\hline 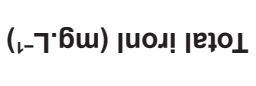 & 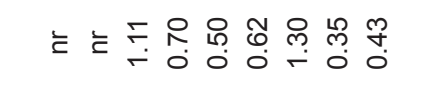 & 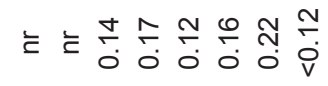 \\
\hline 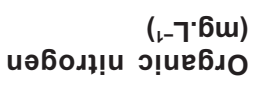 & 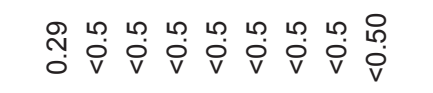 & त̂ \\
\hline 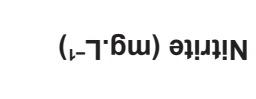 & 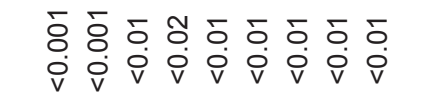 & 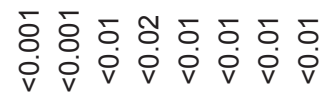 \\
\hline (เ-7'6u) әүеגұ!N & 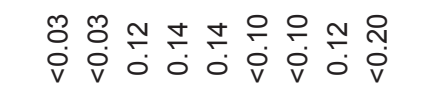 & 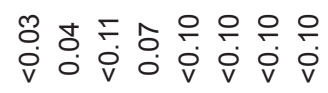 \\
\hline $\begin{array}{r}\left(1-7^{\circ} 6 u\right) \\
\text { uәбодม! |чер|ә!्र }\end{array}$ & 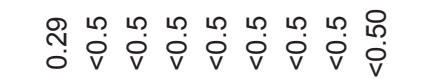 & 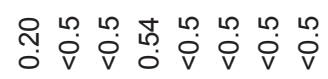 \\
\hline 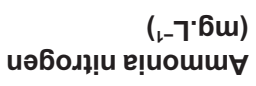 & 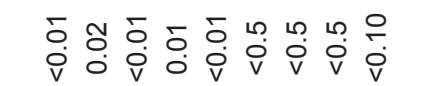 & 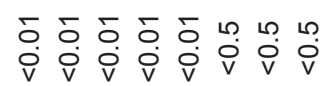 \\
\hline 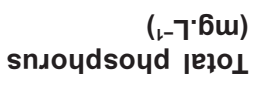 & 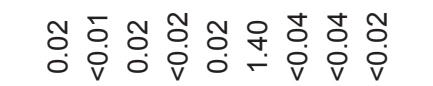 & 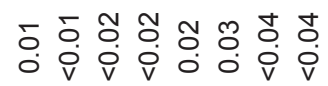 \\
\hline $\begin{array}{r}(1-7 \cdot 6 \mathrm{~m}) \\
\text { әңеydsoydouนO }\end{array}$ & 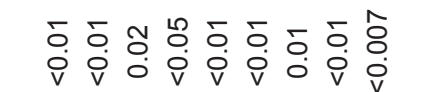 & 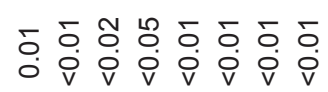 \\
\hline 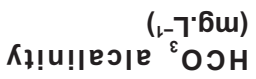 & ণ্ণ & 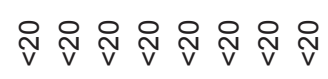 \\
\hline 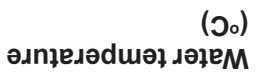 & 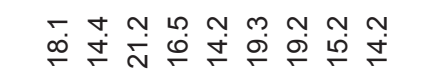 & 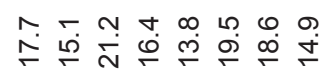 \\
\hline $\mathrm{Hd}^{\mathrm{d}}$ & 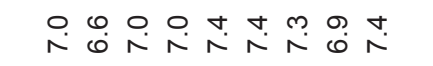 & 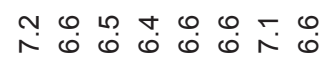 \\
\hline $\begin{array}{r}\left(1-7^{\cdot} 6 u\right) \\
\text { uәбКхо рәл|оss! }\end{array}$ & 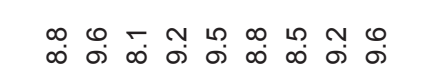 & $\begin{array}{llllllll}m & N & m & \sim & \sigma & 0 & \forall & \infty \\
\infty & \infty & \infty & \infty & \infty & \infty & \infty & \infty\end{array}$ \\
\hline 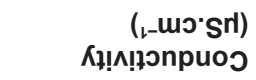 & 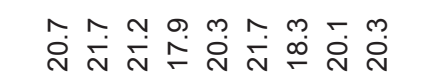 & 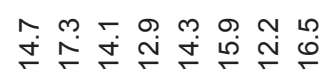 \\
\hline әүер & 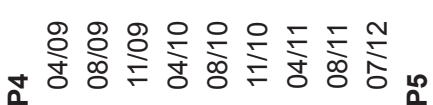 & 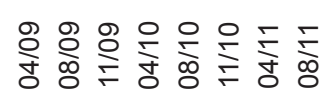 \\
\hline
\end{tabular}


filter technique), total phosphorus (ICP/OES), orthophosphate (ion chromathography), organic nitrogen (ion chromathography), Kjeldahl nitrogen (ion chromathography), nitrate (ion chromathography), nitrite (ion chromathography), ammonia (ion chromathography), iron (ICP/OES), manganese (ICP/OES) and toxicity to Ceriodaphnia dubia (Chronic toxicity - Test with Ceriodaphnia spp). All methods for physical, chemical and microbiological analysis are described in APHA (2005). Ecotoxicological tests were performed according to ABNT (2010). Samplings were according to CETESB (2011).

Samples for aquatic macroinvertebrates analysis were collected with D frame net, exploring all mesohabitats present at the site. Each type of mesohabitat was sampled 3 times at the most. For parameterization of physical diversity of the sampling sites the following types of mesohabitats were distinguished: VEGETATION GROUP - leaf packs, attached and submerged vegetation, logs; LOTIC GROUP - stones (upper and lower surface), slabstone, sandy bottom, gravel; LENTIC GROUP - puddles, pits and pools. The identification level of macroinvertebrates varied according to the group and its importance for the diagnosis.

All laboratory analysis were performed at CETESB, the environmental agency of São Paulo state.

\subsection{Data analysis}

Using macroinvertebrates data the frequencies of occurrence of collected taxa for each site, BMWP (Biological Monitoring Working Party) index adapted by CETEC - Technological Center Foundation of Minas Gerais state - (Junqueira et al., 2000) and Richness (S), as the total number of taxa captured in each site and date of sampling, were calculated.

The mean and the confidence intervals ( $\alpha=0.05)$ for both biotic and abiotic variables were calculated in Microsoft Excel program. Linear correlation coefficient tests $(\alpha=0.05)$ and Principal Component Analysis (PCA) to standardized and centered data, using Past program (Hammer et al., 2001), were performed to verify the relationship between biodiversity indices ( $\mathrm{S}$ and BMWP) and the diversity of mesohabitats, between these same indices and physical and chemical variables, and between those variables and accumulated rainfall in 30, 15 and 7 days from the sampling dates. The $t$ test with a significance level of 0.05 was applied for mean comparisons between some abiotic and biotic variables, also using Past program.

\section{Results}

The study area presents humid tropical climate, with dry season between June and August, and average annual accumulated precipitation of 2,058 mm (Figure 2).

Data showed that the waters of the Paraibuna river are slightly acid and have low content of carbonate (soft water), low values of conductivity (maximum of $21.7 \mu \mathrm{S} . \mathrm{cm}^{-1}$ ), high dissolved oxygen concentrations (at least $7.6 \mathrm{mg} . \mathrm{L}^{-1}$ ) and low concentrations of nutrients, with most values below the limits of quantitation of the analytical methods. Nevertheless, some events of chronic toxicity were observed, including at the reference site (Ipiranga river - P5), and peaks of coliforms concentrations (maximum value of 23,000 CFU.100 $\mathrm{mL}^{-1}$ was obtained in April 2011) at the sampling site located in the Cunha unit (P4) (Table 1).

Conductivity, turbidity, faecal coliforms, iron, phosphorus and nitrate data showed a tendency of water quality gradient improving from upstream to downstream (Figure 3). The highest mean values of these variables, except nitrate, were obtained at Cunha (P4) and the lowest in the Ipiranga river (P5). The $t$ test showed that the mean values of conductivity (P1 x P5: $\mathrm{t}=3.09$ and $\alpha=0.01$; P2 $\times$ P5: $\mathrm{t}=3.47$ and $\alpha=0.005 ; \mathrm{P} 3 \times \mathrm{P} 5: \mathrm{t}=3.14$ and $\alpha=0.007$; P4 x P5: $\mathrm{t}=7.31$ and $\alpha=0.000003)$, turbidity (P1 x P5: $\mathrm{t}=2.31$ and $\alpha=0.04 ; \mathrm{P} 2 \times \mathrm{P} 5$ : $\mathrm{t}=2.71$ and $\alpha=0.02 ; \mathrm{P} 3 \times \mathrm{P} 5: \mathrm{t}=3.11$ and $\alpha=0.01 ; \mathrm{P} 4 \times \mathrm{P} 5: \mathrm{t}=2.24$ and $\alpha=0.04)$ and total iron (P1 x P5: $\mathrm{t}=4.76$ and $\alpha=0.0008$; P2 $\times$ P5: $\mathrm{t}=2.83$ and $\alpha=0.03 ; \mathrm{P} 3 \times \mathrm{P} 5 \mathrm{:} \mathrm{t}=5.32$

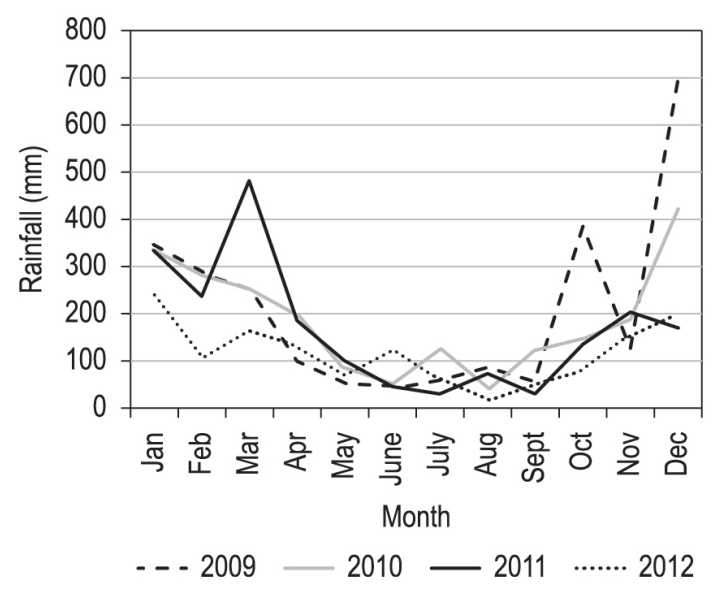

Figure 2. Variation in monthly accumulated rainfall during the study period (2009-2012). 
and $\alpha=0.0005 ; \mathrm{P} 4 \times \mathrm{P} 5: \mathrm{t}=3.40$ and $\alpha=0.01$ ) were significantly lower at the reference site (P5). Concentrations of nitrate $(\mathrm{P} 1 \times \mathrm{P} 5: \mathrm{t}=3.06$ and $\alpha=0.02 ; \mathrm{P} 3 \times \mathrm{P} 5: \mathrm{t}=3.16$ and $\alpha=0.03$; P4 $\times$ P5: $\mathrm{t}=5.94$ and $\alpha=0.004)$ were also significantly lower at P5 when compared to the other sampling sites, with exception of P2. In Cunha (P4), the mean value for Paraibuna river water conductivity were significantly higher $(\mathrm{P} 4 \times \mathrm{P} 1: \mathrm{t}=4.58$ and $\alpha=0.0003 ; \mathrm{P} 4 \times \mathrm{P} 2: \mathrm{t}=5.09$ and $\alpha=0.0002$; P4 x P3: $t=3.72$ and $\alpha=0.002$ ) than the results from other sampling locations. Turbidity and total

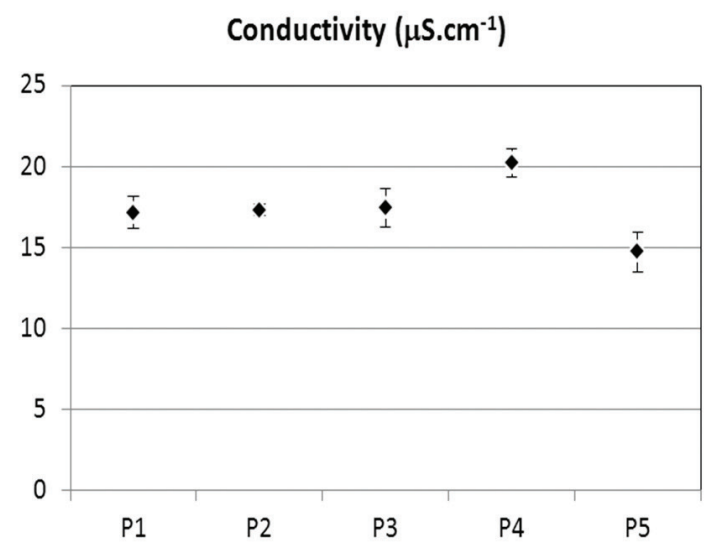

Coliforms (UFC.100 $\mathrm{ml}^{-1}$ )

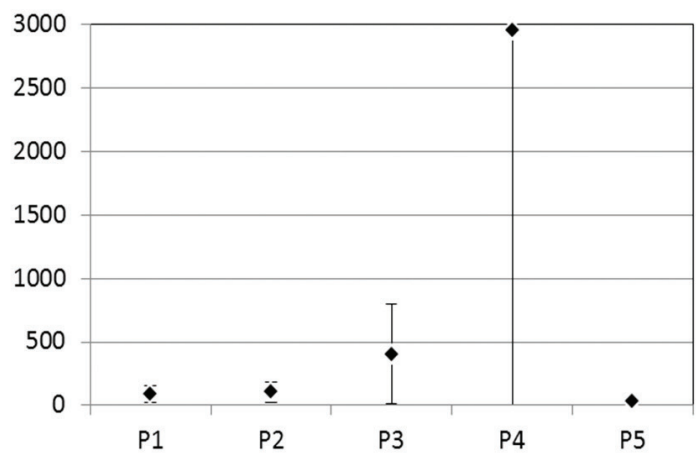

Total phosphorus (mg.t-1)

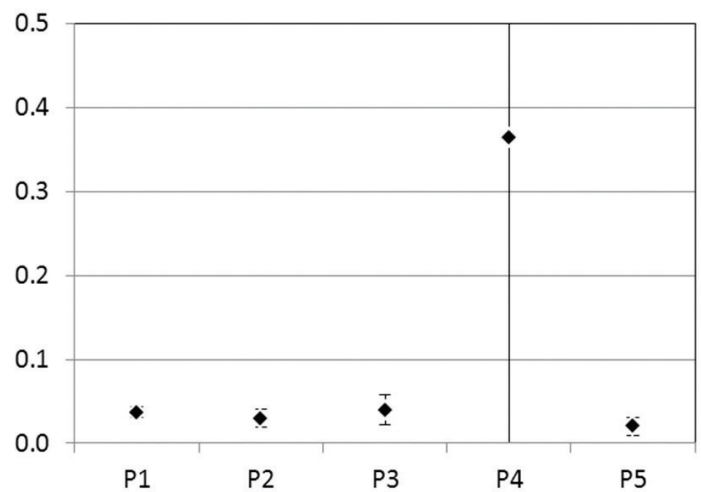

iron are indicators of the existence of active erosional process in the basin and coliforms and nutrients point to the existence of a diffuse source of organic material.

The components 1 and 2 in the PCA (Figure 4) explained $66 \%$ of the total data variation (component $1=40 \%$ and component $2=26 \%$ ). The component 1 correlated better with the variables related to erosional process and diffuse sewage sources in the basin, such as turbidity, iron concentration and coliforms. The component 2 was better correlated with conductivity.
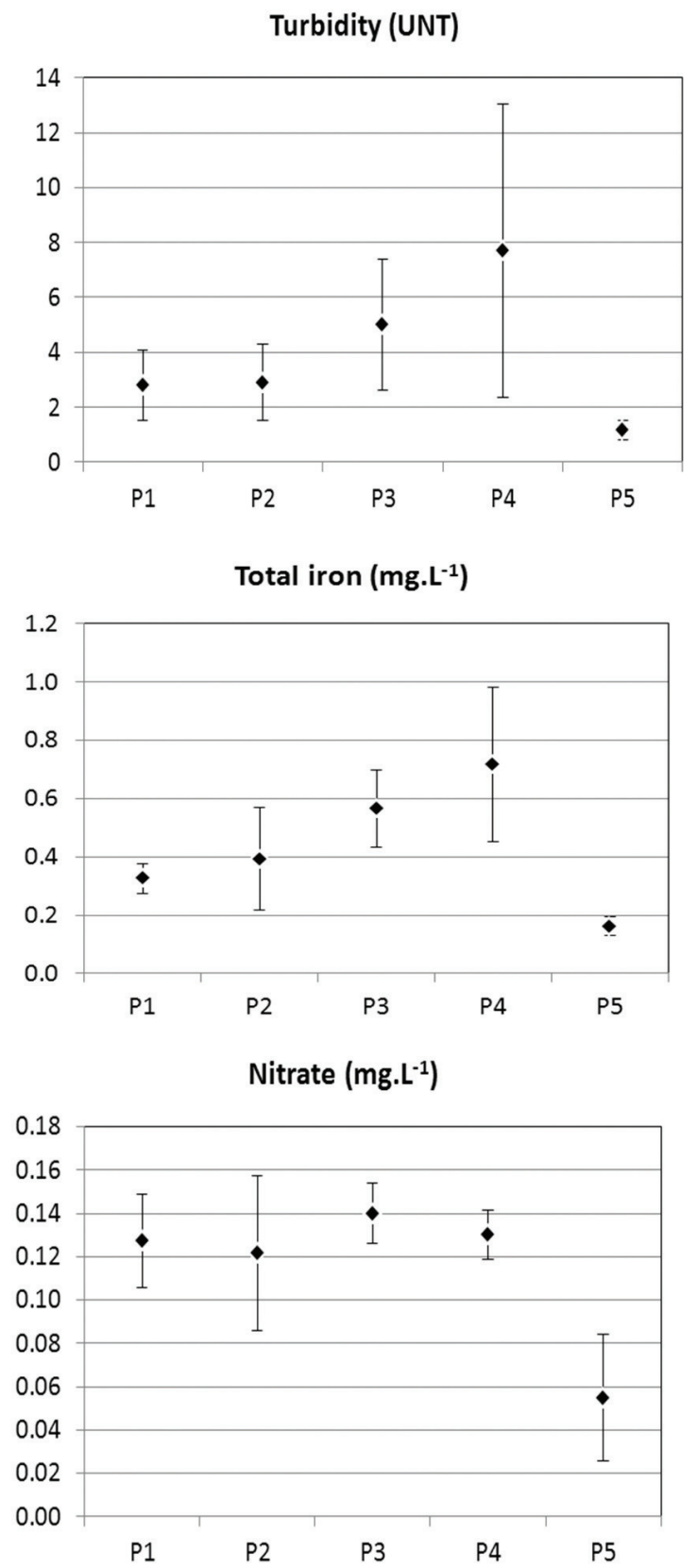

Figure 3. Spatial variation of the mean values of environmental variables, with confidence intervals. 


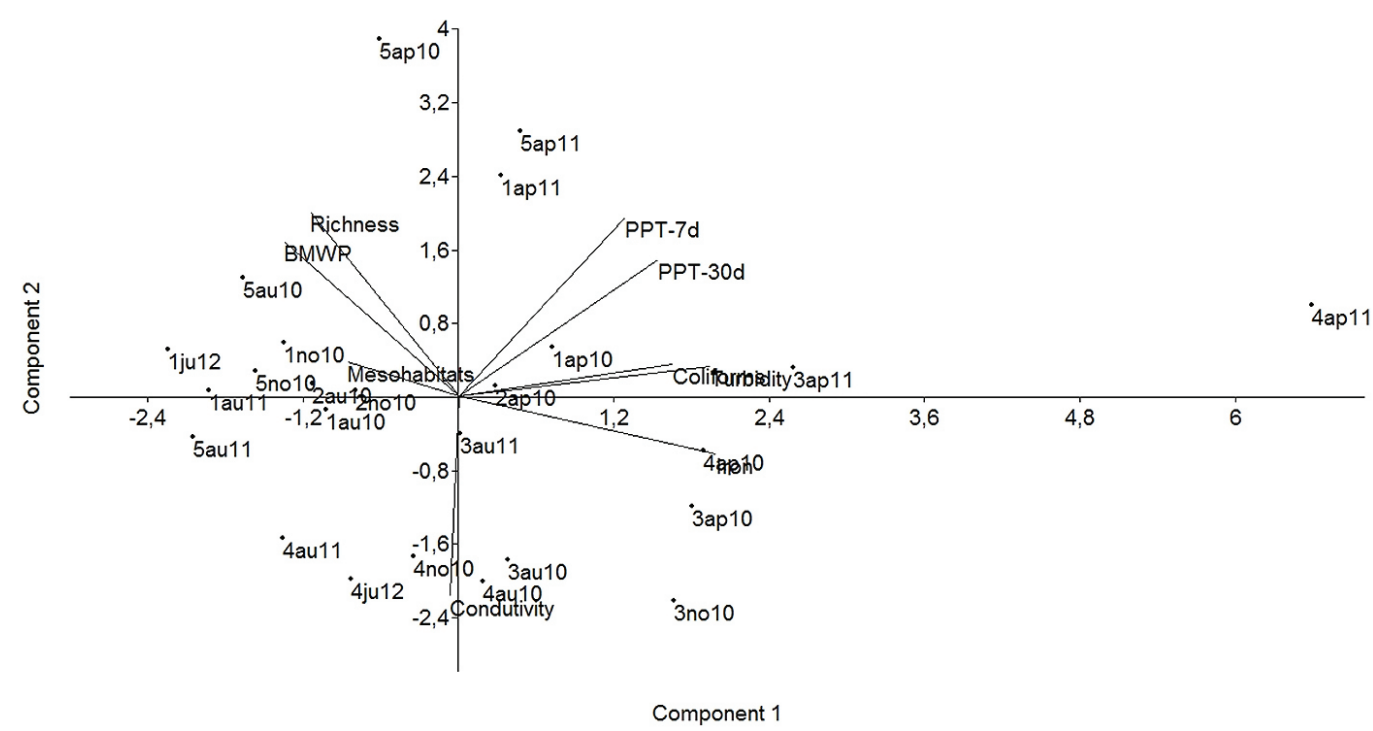

Figure 4. Principal Component Analysis.

These results reflected general patterns, such as the dilution role of rainwater on conductivity in pristine environments and were confirmed by paired Pearson correlation tests (PPT 30 days $\mathrm{x}$ conductivity: $r s=-0.443$ and $\rho=0.004 ; \mathrm{PPT}$ 15 days $x$ conductivity: $r s=-0.408$ and $\rho=0.009$; PPT 7 days $x$ conductivity: $r s=-0.498$ and $\rho=0.001$ ). Furthermore, it could be inferred that the rainfall transports erosional material from surface runoff to the watercourses, including organic waste, especially in the upstream section of the basin (P4), where coliforms (PPT 7 days x coliforms: rs $=0.814$ and $\rho=0.008$ ), iron concentrations (PPT 30 days $\mathrm{x}$ iron: $\mathrm{rs}=0.823$ and $\rho=0.023$ ) and turbidity (PPT 30 days $x$ turbidity: $r s=0.779$ and $\rho=0.013$; PPT 7 days $\mathrm{x}$ turbidity: $\mathrm{rs}=0.725$ and $\rho=0.027$ ) were positively correlated with rainfall. The negative correlation obtained between the rainfall and the value of BMWP index in that stretch (PPT 15 days $x$ BMWP: rs $=-0.735$ and $\rho=0.024$ ) showed that this impact affected its biota.

In the intermediate section of the river (P2 and P3), the lack of correlation between rainfall and the variables related to organic pollution and erosion showed a more buffered environment due to depuration process and protective action of the riparian vegetation of the surrounding area. Further evidence of the environmental service provided by the protected area to Paraibuna river biota was the proximity of the data from its downstream section (P1) and the data from the reference site (P5) (Figure 4). But it should be noted that at P1, the rainfall was related to alterations in turbidity and erosion (Fe) (PPT 30 days x turbidity: rs $=0.775$ and $\rho=0.014 ;$ PPT 7 days: $r s=0.795$ and $\rho=0.010$; PPT 30 days $\mathrm{x}$ iron: $\mathrm{rs}=0.787$ and $\rho=0.036$ ). This section may be under the influence of a rural area, located downstream to P2, drained by a small basin tributary at the right bank of the river. At this site, the river is sided by a road (Vereador Abilio Monteiro de Campos road), parallel to its main course, and the intensification of human activities may threaten the ecological integrity of P1. The Ipiranga river, although fully inserted into the protected area, also showed a correlation between rainfall and surface runoff (PPT $30 \mathrm{~d} x$ iron: $r s=0.884$ and $\rho=0.019$ ), probably due to the existence of another road situated along the riparian area.

The PCA (Figure 4) also showed that the indexes of richness $(S)$ and BMWP, which reflect the state of conservation of aquatic biota, were closely associated with the diversity of mesohabitats, as confirmed by the linear correlation between these variables (mesohabitats $x$ S: $r s=0.459$ and $\rho=0.021$; mesohabitats $\mathrm{x}$ BMWP: $\mathrm{rs}=0.481$ and $\rho=0.015)$. These two metrics were negatively related to indicators of erosional processes (BMWP $\mathrm{x}$ Fe: $\mathrm{rs}=-0.455$ and $\rho=0.011 ; S \mathrm{x}$ Fe: $\mathrm{rs}=0.409$ and $\rho=0.025$ ), demonstrating its sensitivity to impacts of this kind.

Regarding macroinvertebrates, 88 taxa were observed, 33 of which had frequencies of occurrence $\leq 25 \%$ at the five sites sampled. Some species were restricted to one place or even one mesohabitat, such as Chaoboridae larvae in pits at P1. On the 
Table 2. Appearance frequency of macroinvertebrates taxa in the five sampling sites.
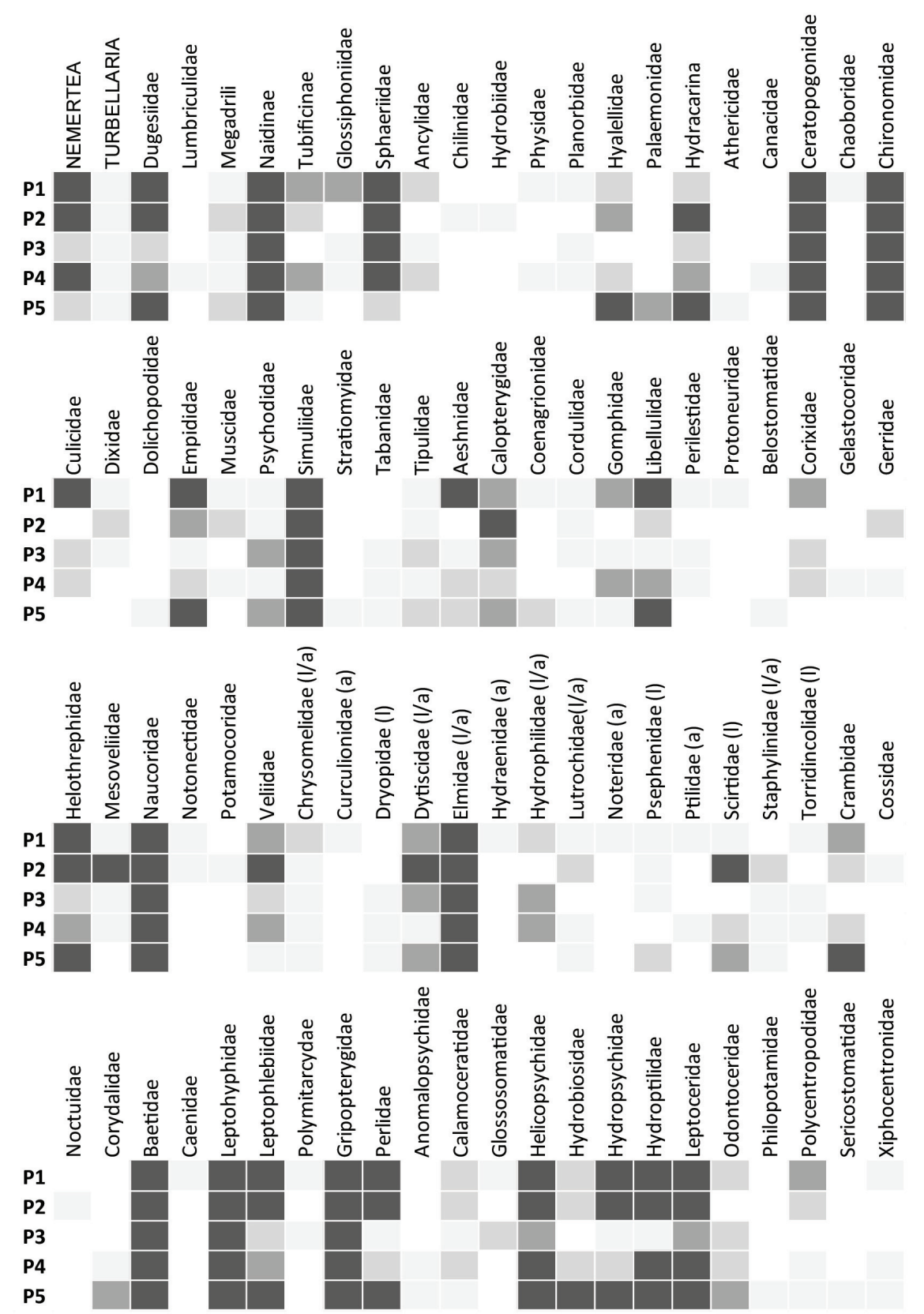

I = larvae; $\mathrm{a}=$ adult

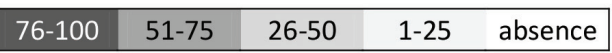

other hand, nine taxa were present in all sites at frequencies above $75 \%$ (Table 2).

The sensitivity of taxa richness and BMWP index to mesohabitats diversity and to the negative effects of erosion, and in some sampling sites, also to rainfall and turbidity was especially clear in the results from the section of the Paraibuna river at Lajeado (P3), that presented the smaller average diversity of mesohabitats $(\mathrm{P} 3 \times \mathrm{P} 1: \mathrm{t}=-3.86$ and $\alpha=0.004 ; \mathrm{P} 3 \times \mathrm{P} 4: \mathrm{t}=-5.28$ and $\alpha=0.005$;
P3 x P5: $\mathrm{t}=-3.81$ and $\alpha=0,01$ ) (Figure 5) and the lower total richness $(\mathrm{P} 3 \times \mathrm{P} 1: \mathrm{t}=-4.03$ and $\alpha=0.001 ; \mathrm{P} 3 \times \mathrm{P} 2: \mathrm{t}=-2.91$ and $\alpha=0.02 ; \mathrm{P} 3 \times \mathrm{P} 5$ : $\mathrm{t}=-4.33$ and $\alpha=0.0007$ ) (Figure 6). Moreover, its biota was composed mostly of taxa with low frequency of occurrence $(\mathrm{FO} \leq 50 \%)$. On the other hand, the biota from the downstream sections of the Paraibuna river (P1 and P2) and Ipiranga river (P5) were formed mainly by groups that occurred with some constancy (FO >75\%) (Table 2). At these 
locations the highest mean richness $(\mathrm{S})$ were also obtained (Figure 6). The section of the Paraibuna river at Cunha (P4), despite having presented the highest diversity average of mesohabitats, was composed of taxa with low frequency (Table 2) and did not reach the highest total taxa richness, as expected.

The mean values of the BMWP index showed a similar scenario (Figure 7). The value obtained at the reference site (P5) was significantly higher than those from the other locations, with exception of P1 (P5 x P2: $\mathrm{t}=2.56$ and $\alpha=0.03$; P5 $x$ P3: $\mathrm{t}=4.72$ and $\alpha=0.0003$; P5 $\times$ P $4: \mathrm{t}=3.43$ and

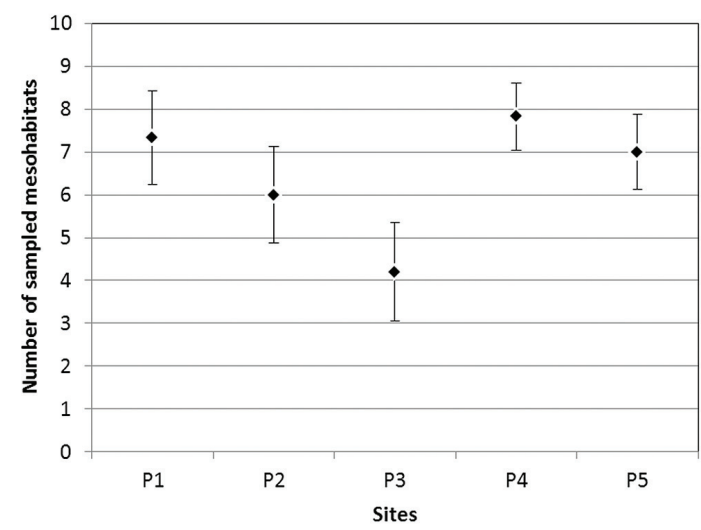

Figure 5. Average mesohabitats sampled in different sites and their confidence intervals.

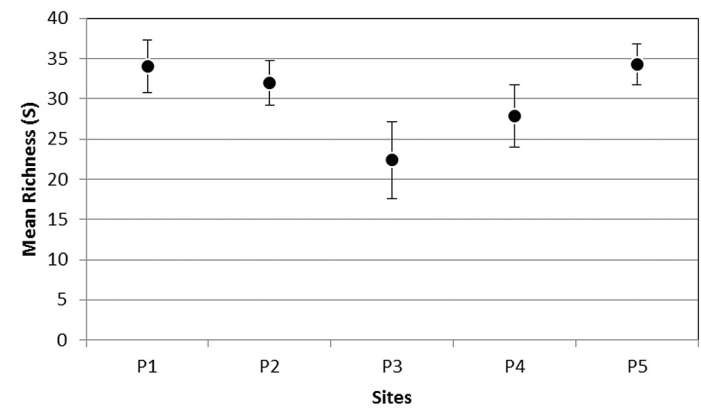

Figure 6. Spatial variation of mean Richness $(S)$ and their confidence intervals.

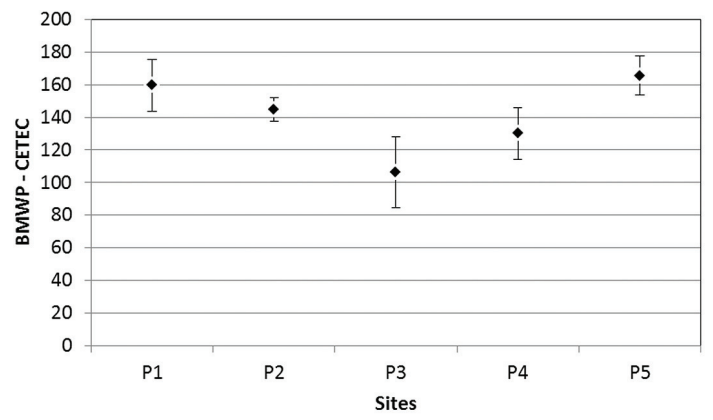

Figure 7. Spatial variation of BMWP - CETEC index and their confidence intervals. $\alpha=0.004)$. Considering the quality ranges proposed in CETEC's adaptation (Junqueira et al., 2000), all the locations presented Excellent quality, with the exception of two occasions when the biota of P3 indicated Good (Apr/10) and Regular quality (Nov/10).

\section{Discussion}

Despite the good quality of the Paraibuna river waters, as indicated by the majority of the physical and chemical parameters analyzed, deviations from the values obtained in the reference area, chosen to represent the natural condition, were observed. The establishment of a reference area is recommended by CONAMA Resolution 357/05 (Brasil, 2005, p. 58) to guide parameter values for special class waters, by definition as those intended for the "[...] preservation of aquatic environments in Conservation Areas of Strict Protection". All sections of the Paraibuna river had higher values for conductivity, turbidity, coliform, iron, total phosphorus and nitrate in the water than the Ipiranga river. The upstream section of Paraibuna river in Cunha (P4) showed the greatest variation compared to the reference data and, although located $20.9 \mathrm{~km}$ downstream, the section in Lajeado (P3) also presented higher variation in colimetric assays, iron and turbidity than the other downstream sites. Variations in nutrient and colimetric assays reflect the land use by small farms with animal husbandry, such as cattle, sheep and chicken. The highest values of coliforms were related to the occurrence of rainfall that carries surface deposits of waste and can cause septic tanks leaking. The gradient observed in Paraibuna river shows the recovery of the water quality, although partial, in the section of the river between the Cunha unit, which receives directly impacts of the human occupation in its headwaters, and the area of Salto Grande in Santa Virginia unit. At this site the river can be under the influence of a small drainage basin located on its right bank, on a portion not inserted in the protected area, downstream to P2. These results highlight the buffering role and consequent ecosystem services of the protected area to the Paraibuna river water quality, used not only as scenic beauty and ecotourism, but also for public supply and energy production in the region.

The toxicity events observed, including at the Ipiranga river, can be related to the release of oil, polyphenols and terpenes by decomposition of eucalyptus leaves, an invasive species widely distributed in both units and in the headwaters 
area of the Paraibuna river. These substances, used by plants as a defense mechanism to herbivory and to the action of bacteria and fungi, have proven toxic effect to aquatic organisms (Canhoto and Laranjeira, 2007; Graça et al., 2002). However, it should be noted that the events of toxicity observed in the laboratory were not accompanied by local macrofauna losses in biodiversity.

Regarding the aquatic macroinvertebrates data, the section in the Paraibuna river at Lajeado (P3) and in the Cunha unit (P4) presented the lowest richness, composed of taxa with less frequent occurrence, and obtained the lowest values for the BMWP index. These results reflected the instability and the sensitivity of those sites to changes in water quality, and in $\mathrm{P} 3$, also the low mesohabitats diversity. The influence of spatial heterogeneity on the biodiversity of macrofauna highlighted in this work by the richness and BMWP indices corroborates one of the ecological principles of Thienemann (Vinson and Hawkins, 1998). These indices were also sensitive to the effects, even subtles, of erosion and input of organic matter observed in the basin, validating them to be used in the biomonitoring of these environments, as well as the classifications employed for distinguishing mesohabitats.

In the Ipiranga and Paraibuna rivers, within Santa Virginia unit, the presence of the fish pirapitinga-do-Paraíba or pirapitinga-do-sul (Brycon opalinus), an endangered species (São Paulo, 2010) depends on the recovery of its habitats (Rosa and Lima, 2008) to continue to exist. According to Gomiero and Braga (2007), low turbidity, high concentration of dissolved oxygen in the river and the preservation of the forest, which prevents siltation and offers food to the species, are environmental requirements for its occurrence. Turbidity was one of the variables affected by land use in the headwaters area of the Paraibuna river. If this impact intensifies, the turbidity values can be altered in the region of Salto Grande, where a population of pirapitinga-do-sul lives, threatening its survival and other species populations of equal sensitivity.

Although the existence of an endangered species in one portion of the Paraibuna river can be used as a tool to biodiversity conservation, many ecologists concerned with the preservation of riparian environments have defended that this approach, widely used in the conservation of terrestrial biota, should be abandoned. To Strayer (2006), for example, an approach that considers the whole watershed as the protected area and uses quality criteria for the protection of aquatic life in monitoring the water resources that flows through protected areas are strategies that can protect aquatic biodiversity more effectively. Furthermore, Corkum (1999) points out the importance of not dissociating the conservation of the river to the surrounding terrestrial environment that, if is not encompassed in the protected areas, should at least be subject to public administration to ensure that human activities follow environmentally sustainable practices.

In Brazil, the approach adopted for the selection of protected areas has been the conservation of representative areas of its major biomes, covering its flora, fauna and aquatic ecosystems. Unfortunately, in some situations the conservation of the faunal and floristic patrimony of aquatic systems has not been considered at the time of the delimitation of the areas, exposing that biota to degradation by anthropogenic activities located upstream.

For the full protection of the headwaters of the Paraibuna river, the addition of the unprotected area upstream of the Cunha unit would represent an increase of $124.30 \mathrm{~km}^{2}$ to the protected area, which corresponds to almost double its current area. As for the protection of the area on the right bank of the river, in the section next to P1 and P2 in Santa Virginia unit, it would represent an increase of $58.42 \mathrm{~km}^{2}$, or $33 \%$ of its current area. Instead of adding these areas to the protected area, a more plausible solution would be the creation of a Sustainable Use Area, as Environmental Protection Areas (EPA), which would allow greater control over the land use in the headwaters of the Paraibuna river.

On the other hand, Brazilian legislation (CONAMA 357/05) (Brasil, 2005) already provides quality criteria for waters comprised in Strict Protection Areas, classifying them as special class. It is up to governmental agencies responsible for monitoring water quality to adopt biodiversity conservation as a quality target, using appropriate indicators and inserting monitoring sites in water bodies that run through protected areas, especially those that have vulnerable upstream region.

\section{Conclusion}

Anthropogenic activities upstream the PESM, and probably also in areas located on the right bank of the Santa Virgínia unit, have negative effects on the ecological quality of the Paraibuna river and can jeopardize its aquatic biodiversity. Those effects can be minimized if the currently adjacent 
populated areas were converted into Environmental Protection Areas that would allow the maintenance of the existing occupation and regulate the land use, especially in the implementation of measures of erosion control and waste water treatment. Areas of interest to the protection of the terrestrial wildlife, with significant vegetal cover, could be attached to existing Conservation Areas of Strict Protection.

Furthermore, public authorities responsible for the administration of the road that follows the river banks must provide a drainage system for rainfall waters, which contribute to the transportation of particulate matter into the river.

The effectiveness of the control measures should be monitored with aquatic life protection indicators that can ensure the quality of the river, not only for the survival of endangered species but the entire biota.

\section{Acknowledgements}

The authors thanks to all professionals of the Department of Environmental Analysis of CETESB who directly or indirectly contributed to the data collection, especially to the technician Osvaldo A. da Silva, loyal companion in the samplings, and $\mathrm{Dr}$ Marta C. Lamparelli and biologist Adriana C. C. R. Deus for reviewing the English version. Thanks to biologist Dr João Carlos C. Milanelli, whose initiative has enabled our involvement with the conservation issue and development of this project. To Fundação Florestal and Instituto Florestal for their support in developing this project.

\section{References}

Associação Brasileira de Normas Técnicas - ABNT. 2010. NBR 13373. Ecotoxicologia aquática - Toxicidade crônica - Método de ensaio com Ceriodaphnia spp (Crustacea, Cladocera). Rio de Janeiro.

Allan, JD. 2004. Landscapes and riverscapes: the influence of land use on stream ecosystems. Annual Review of Ecology, Evolution, and Systematics, vol. 35, p. 257-284. http://dx.doi.org/10.1146/annurev. ecolsys.35.120202.110122

ALLAN, JD. and FLECKER, AS. 1993. Biodiversity conservation in running waters. BioScience, vol. 43, no. 1, p. 32-43. http://dx.doi.org/10.2307/1312104

American Public Health Association - APHA. 2005. Standard methods for the examination of water and wastemaster. 21th ed. Washington.

Brasil. 2000. Lei n. 9.985, de 18 de julho de 2000. Diário Oficial da União (da) República Federativa do Brasil, Poder Executivo, Brasília, DF, 19 jul. 2000. p. 1.

Brasil. Ministério do Meio Ambiente - MMA. 2003. Instrução Normativa n. 3, de 27 de maio de 2003.
Diário Oficial da União (da) República Federativa do Brasil, Poder Executivo, Brasília, DF, 28 maio 2003. p. 88.

Brasil. Ministério do Meio Ambiente - MMA. 2004. Instrução Normativa n. 5, de 21 de maio de 2004. Diário Oficial da União (da) República Federativa do Brasil, Poder Executivo, Brasília, DF, 28 maio 2004. p. 136.

Brasil. 2005. Resolução CONAMA n. 357, de 17 de março de 2005. Diário Oficial da União (da) República Federativa do Brasil, Poder Executivo, Brasília, DF, 18 mar. 2005. p. 58.

CANHOTO, C. and LARANJEIRA, C. 2007. Leachates of Eucalyptus globulus in intermittent streams affect water parameters and invertebrates. International Review of Hydrobiology, vol. 92, no. 2, p. 173-82. http://dx.doi.org/10.1002/iroh.200510956

Companhia Ambiental do Estado de São Paulo - CETESB. 2011. Guia nacional de coleta $e$ preservação de amostras. água, sedimento, comunidades aquáticas e efluentes líquidos. Brasília. p. 325.

CORKUM, LD. 1999. Conservation of running waters: beyond riparian vegetation and species richness. Aquatic Conservation: Marine and Freshwater Ecosystems, vol. 9, no. 6, p. 559-564. http://dx.doi.org/10.1002/ (SICI)1099-0755(199911/12)9:6<559::AIDAQC384>3.0.CO;2-\#

GOMIERO, LM. and BRAGA, FMS. 2007. Reproduction of Pirapitinga do Sul (Brycon opalinus Cuvier, 1819) in the Parque Estadual da serra do Mar - Núcleo Santa Virgínia, São Paulo, Brazil. Brazilian Journal of Biology, vol. 67, no. 3, p. 541549. PMid:18094839. http://dx.doi.org/10.1590/ S1519-69842007000300021

GRAÇA, MAS., POZO, J., CANHOTO, C. and ELOSEGI, A. 2002. Effects of Eucalyptus plantations on detritus, decomposers, and detritivores in streams. The Scientific World Journal, vol. 2, p. 1173-1185. PMid:12805976. http://dx.doi.org/10.1100/ tsw.2002.193

HAMMER, Ø., HARPER, DAT. and RYAN, PD. 2001, PAST: Paleontological statistics software package for education and data analysis. Palaeontologia Electronica, vol. 4, no. 1, 9 p.

JUNQUEIRA, MV., AMARANTE, MC., DIAS, CFS. AND FRANÇA, ES. 2000. Biomonitoramento da qualidade das águas da bacia do Alto rio das Velhas (MG/Brasil) através de macroinvertebrados. Acta Limnologica Brasiliencia, vol. 12, p. 73-87.

ROSA, RS. and LIMA, FCT. 2008. Peixes. In MACHADO, ABM., DRUMMOND, GM. and PAGLIA, AP., ed. Livro vermelho da fauna brasileira ameaçada de extinção. 1. ed. Brasília: MMA. 278 p. Biodiversidade 19. 
ROSENBERG, DM. and RESH, VH. 1993. Freshwater biomonitoring and benthic macroinvertebrates. New York: Chapman \& Hall. 504 p.

SÃO PAULO. 2004. Resolução SMA n. 48, de 21 de setembro de 2004. Available from: <http://www.ibot. sp.gov.br/pesquisa_cientifica/restauracao_ecologica/ resolu\%C3\%A7\%C3\%A3o_\%20sma48.pdf>. Acess in: may 2013.

SÃO PAULO. 2006. Parque Estadual da Serra do Mar: plano de manejo. São Paulo: Secretaria de Estado do Meio Ambiente. 441 p. vol. 1. Available from: $<$ http://www.iflorestal.sp.gov.br/Plano_de_manejo/ PE_SERRA_MAR/Plano_de_Manejo_Pe_Serra_ do_Mar.pdf>. Acess in: july 2013.

SÃO PAULO. 2010. Decreto Estadual n. 56.031, de 20 de julho de 2010. Available from: <http:// www.ambiente.sp.gov.br/wp-content/uploads/
decreto/2010/2010_Dec_56031.pdf>. Acess in: mar. 2013.

SÃO PAULO. 2013. Parque Estadual da Serra do Mar. Available from: Disponível em: <http://florestal. sp.gov.br/unidades-de-conservacao/parquesestaduais/parques-estaduais/>. Acess in: july 2013.

STRAYER, DL. 2006, Challenges for freshwater invertebrate conservation. Journal of the North American Benthological Society, vol. 25, no. 2, p. 271-287. http://dx.doi.org/10.1899/08873593(2006)25[271:CFFIC]2.0.CO;2

VINSON, MR. and HAWKINS, CP. 1998, Biodiversity of stream insects: variation at local, basin and regional scales. Annual Review of Entomology, vol. 43, p. 271293. PMid:15012391. http://dx.doi.org/10.1146/ annurev.ento.43.1.271

Received: 20 August 2013

Accepted: 19 May 2014 